\title{
Composición Corporal y Somatotipo de Mujeres de Entre 16 a 18 Años: Efecto de un Programa Acuático-Aeróbico en el Mejoramiento de la Fuerza Muscular
}

\author{
Body Composition and Somatotype in Women between 16 and 18 Years of Age: \\ Effect of an Aquatic-Aerobics Program on the Improvement of Muscular Strength
}

\author{
Anita Pino-Reynals; ; Omar Espinoza-Navarro**; Miguel De Arruda**** \& Carlos Urizar-Araya ${ }^{* * * * *}$
}

PINO-REYNALS, A.; ESPINOZA-NAVARRO, O.; DE ARRUDA, M. \& URIZAR-ARAYA, C. Composición corporal y somatotipo de mujeres de entre 16 a 18 años: efecto de un programa acuático-aeróbico en el mejoramiento de la fuerza muscular. Int. J. Morphol., 33(4):1213-1219, 2015.

RESUMEN: Las actividades acuáticas son una clara alternativa a los programas de reforzamiento muscular terrestre. El objetivo de este trabajo fue determinar el efecto de un programa de ejercicios acuáticos-aeróbicos sobre pliegues y diámetros corporales y en el mejoramiento de la fuerza muscular de mujeres jóvenes. Doce mujeres de entre 16 a 18 años, sedentarias, seleccionadas intencionalmente fueron sometidas a un programa de ejercicios acuáticos de 18 sesiones, desarrollados en la Piscina Olímpica Municipal de la ciudad de Arica, Chile. Los resultados muestran que las jóvenes presentan un IMC de sobrepeso, con un somatotipo predominantemente endomórfico. Los pliegues corporales de muslo anterior y pantorrilla disminuyen significativamente al final de la aplicación del programa, igual comportamiento expresa la sumatoria de ellos ( $\sum 7$ : de 167,06 $\pm 8,71 \mathrm{~mm}$ a $145,23 \pm 8,9 \mathrm{~mm}$ en pre-test). La fuerza muscular se incrementa significativamente en los músculos pectoral mayor, latísimo del dorso y bíceps braquial. Sin embargo este incremento es más significativo ( $\mathrm{p}<0,001)$ en la sumatoria de los músculos evaluados ( $\sum 7$ : de 137,72 $\pm 5,27 \mathrm{~kg}$ a $158,67 \pm 6,48 \mathrm{~kg}$ al finalizar el programa). Se concluye que el programa de ejercicios acuáticos-aeróbicos aplicado a las jóvenes, tiene un efecto positivo, con aumento significativo en la fuerza muscular y una disminución significativa en pliegues cutáneos. La aplicación de este tipo de programas, otorga múltiples beneficios para la salud y la mejora de la calidad de vida.

PALABRAS CLAVE: Ejercicio Acuático; Fuerza muscular; Natación; Chile.

\section{INTRODUCCIÓN}

Las actividades acuáticas se desarrollan en variados tipos de prácticas motrices que se realizan de muy diversas maneras en el agua, orientados a objetivos educativos, competitivos, utilitarios, recreativos, preventivos, terapéuticos y de entrenamiento-mantenimiento (Barbosa et al., 2009). Los programas de actividades acuáticas-aeróbicas, son una clara alternativa a los ejercicios realizados en ambiente terrestre (Pantelic et al., 2013). Las múltiples cualidades del medio acuático proporcionan grandes ventajas para desarrollar la fuerza. Se suelen utilizar programas de movimientos variados y de gran amplitud para no sobrecargar las articulaciones. Se favorece el enriquecimiento motor, se evitan las lesiones y se obtiene un trabajo de tonificación más global. Evidentemente, la ejercitación en el agua es una práctica que reúne las ventajas y los requisitos necesarios para ser considerada como saludable (Pöyhönen et al., 2002). Algunas de estas ventajas son: hipogravidez, presión hidrostática, resistencia al movimiento, aumento de la intensidad del ejercicio, mejor equilibrio muscular y facilitación de la termorregulación. Se planifican actividades que resaltan y recuperan para su práctica habitual posiciones verticales y horizontales en inmersión total o parcial. Son considerados una herramienta muy útil en los procesos de rehabilitación. Además de los beneficios a nivel orgánico, también consi-

\footnotetext{
Centro de Formación Técnica (CFT) de Tarapacá, Escuela Superior de Educación y Humanidades, Arica, Chile.

** Profesor Titular, Laboratorio Biología de la Reproducción y Desarrollo, Departamento de Biología, Facultad de Ciencias, Universidad de Tarapacá, Arica, Chile.

**** Facultad de Educación Física, Universidade Estadual de Campinas (UNICAMP), Campinas, Brasil.

${ }^{* * * * *}$ Kinesiólogo, Departamento de Salud Municipal (DESAMU), Unidad de Rehabilitación, Centro de Rehabilitación Familiar, Centro de Salud Familiar (CESFAM) Víctor Bertín Soto, Arica, Chile.
} 
gue ventajas psicológicas (mejora de la autoestima, el auto concepto, etc.), consolidando en forma más efectiva hábitos estables hacia la práctica de la actividad física (World Health Organization, 2004; Becker, 2009).

A través de los programas de ejercicios acuáticosaeróbicos, se pretende desarrollar la fuerza muscular, que es la capacidad de vencer $\mathrm{u}$ oponerse ante una resistencia externa mediante tensión muscular (Colado \& Triplett, 2009). Con un entrenamiento de fuerza también se incrementa la masa magra y se disminuye la grasa. El músculo es metabólicamente más activo y aumenta la tasa metabólica, la oxidación de grasa y el consumo de calorías.

El objetivo del estudio fue determinar el efecto de un programa de ejercicios acuático-aeróbicos sobre el grado de variación en el desempeño de la fuerza muscular y en los antecedentes antropométricos de peso, estatura, índice de masa corporal (IMC), perímetros musculares, pliegues cutáneos y en el somatotipo de jóvenes sedentarias de entre 16 y 18 años.

\section{MATERIAL Y MÉTODO}

El presente estudio es de tipo descriptivo-exploratorio comparativo. Con un diseño pre-experimental, sin grupo control. El universo de esta investigación fue de 55 mujeres sedentarias entre 16 y 18 años que asisten regularmente a las actividades acuáticas que se desarrollan en la Piscina Olímpica de Arica. La muestra utilizada es de tipo intencionada y fue constituida por 12 mujeres que no participaban de actividades físicas sistemáticas de mejoramiento de fuerza muscular y que no poseían patología o contraindicaciones para realizar actividad física o ejercicio, representando a un $22 \%$ de la población investigada. Los protocolos ocupados de Consentimiento Informado y Asentimiento de las jóvenes, fueron aprobados por el Comité de Ética y Bioética de la Universidad de Tarapacá.

Las alumnas fueron pesadas y medidas para realizar el cálculo del IMC. Los valores obtenidos se analizaron por las "Normas de evaluación nutricional en niños chilenos de entre 16 a 18 años (Unidad de Nutrición del Ministerio de Salud \& Consejo Asesor en Nutrición, 2004). Posteriormente se evaluaron los pliegues, perímetros y diámetros corporales siguiendo las indicaciones de "International Society for the Advancement of Kinanthropometry" (Marfell-Jones et al., 2006; Díaz \& Espinoza-Navarro, 2012).

Para medir el peso corporal se utilizó balanza digital "Camry" (precisión de 100 g). La talla se obtuvo con estadiómetro de pared (precisión de $5 \mathrm{~mm}$ ). Los perímetros corporales se midieron con cinta antropométrica Sanny Medical (precisión de $1 \mathrm{~mm}$ ). Los pliegues grasos fueron obtenidos con cáliper Harpenden. Estas mediciones no invasivas se ejecutaron en la Piscina Olímpica de Arica-Chile, cada medición se repitió dos veces, el promedio de tiempo ocupado para cada sujeto evaluado fue de $10 \mathrm{~min}$.

Posteriormente las jóvenes fueron sometidas a un programa de 18 clases de ejercicios acuático-aeróbicos, para el desarrollo de la fuerza muscular, con una frecuencia de trabajo de tres sesiones por semana y de 45 min de duración por sesión. El tiempo total ocupado en el programa fue de seis semanas.

Todas las mediciones y los antecedentes antropométricos se realizaron al comienzo del programa (pretest) y al final de la intervención de ejercicios acuáticoaeróbicos (post-test). De igual manera, se midió la fuerza muscular utilizando la prueba de una repetición máxima, 1 RM (Espinoza-Navarro \& Valle, 2014). Se consideraron en estas mediciones a los músculos: pectoral mayor, glúteo máximo, recto abdominal, abdominal corto, latísimo del dorso, bíceps braquial y tríceps braquial. Se controló la frecuencia cardiaca máxima para manejar la intensidad de los ejercicios del programa acuático al $70 \%$ de esfuerzo, además se midió la escala de esfuerzo percibido (Edwards, 1996; Colado \& Triplett, 2009) y la intensidad del ejercicio con la escala psicofísica de Borg (Brunetto et al., 2002), se registró la temperatura del agua como antecedente adicional (Moreno \& Gutiérrez, 1998). Las mediciones antropométricas fueron supervisadas por un profesional acreditado como evaluador nivel II por la International Society for the Advancement of Kinanthropometry (ISAK, 2001).

Análisis estadístico. Todos los datos obtenidos fueron procesados con el paquete estadístico SPSS 19. Utilizando los estadísticos descriptivos de Media y Desviación Estándar (Media_DE). Luego se aplicó la prueba de t Student como estadístico paramétrico. El nivel de significancia fue de $\mathrm{p}<0,05$.

\section{RESULTADOS}

La Tabla I, muestra la Media $\pm D E$ de la composición corporal de IMC, la densidad corporal, el porcentaje de grasa corporal y el índice cintura/cadera $(\mathrm{Ci} / \mathrm{Ca})$. Se observa que el IMC en pre-test y post-test indican que la población en estudio, se encuentra con sobrepeso. Al finalizar el programa se observa una disminución en el índice $\mathrm{Ci} / \mathrm{Ca}$ y en el porcentaje de grasa corporal, sin embargo estos valores no son estadísticamente significativos. 
En la Tabla II se presenta la Media \pm DE, de los pliegues y la sumatoria de ellos $\left(\sum 7\right)$. En el post-test se observa una disminución significativa en los pliegues de muslo anterior, pantorrilla y en la sumatoria de todos los pliegues $(\mathrm{p}<0,001$ y $\mathrm{p}<0,05)$.

La Tabla III, muestra los resultados comparativos de los perímetros y diámetros corporales obtenidos en las jóvenes mujeres. No se observan diferencias significativas entre el pre y post-test (Media $\pm \mathrm{DE})$.

En la Tabla IV, se observa que el somatotipo (Media \pm DE), de las jóvenes evaluadas en el pre y en post-test es predominantemente endomórfico (Carter, 2002). No se observan diferencias significati-

Tabla I. Valores comparativos pre-test y post-test de las jóvenes en estudio para el peso, talla, índice de masa corporal (IMC), densidad corporal, porcentaje de grasa corporal e índice cintura/cadera $(\mathrm{Ci} / \mathrm{Ca})$.

\begin{tabular}{lll}
\hline $\begin{array}{l}\text { Mujeres 16 - 18 años } \\
\mathbf{n}=\mathbf{1 2}\end{array}$ & $\begin{array}{l}\text { Pre-Test } \\
(\text { Media } \pm \text { DE) }\end{array}$ & $\begin{array}{l}\text { Post-Test } \\
\text { (Media } \pm \text { DE) }\end{array}$ \\
\hline Peso $(\mathrm{kg})$ & $61,25 \pm 11,61$ & $62,16 \pm 11,75$ \\
Talla $(\mathrm{m})$ & $1,61 \pm 5,1$ & $1,61 \pm 5,2$ \\
IMC & $27,8 \pm 3,9$ & $27,6 \pm 3,5$ \\
Índice Ci/Ca & $0,81 \pm 0,08$ & $0.72 \pm 0,07$ \\
Grasa corporal $(\%)$ & $28,12 \pm 4,05$ & $25,88 \pm 3,75$ \\
Densidad corporal $(\mathrm{g} / \mathrm{cc})$ & $1,03 \pm 0,01$ & $1,04 \pm 0,01$ \\
\hline
\end{tabular}

Media \pm Desviación Estándar (DE).

Tabla II. Valores de los pliegues corporales y Sumatoria de 7 pliegues $\left(\sum 7\right)$, al inicio (pre test) y al final (post test) de la aplicación del programa de ejercicios acuáticos-aeróbicos en las jóvenes de Arica, Chile.

\begin{tabular}{lcc}
\hline $\begin{array}{l}\text { Mujeres 16 - 18 años } \\
\mathbf{n}=\mathbf{1 2}\end{array}$ & $\begin{array}{c}\text { Pre-Test } \\
\text { (Media } \pm \text { DE) }\end{array}$ & $\begin{array}{c}\text { Post-Test } \\
\text { (Media } \pm \text { DE) }\end{array}$ \\
\hline Tríceps & $19,83 \pm 4,14$ & $17,75 \pm 4,29$ \\
Bíceps & $13,50 \pm 5,25$ & $12,00 \pm 4,5$ \\
Subescapular & $22,75 \pm 8,37$ & $19,16 \pm 8,37$ \\
Abdominal & $29,66 \pm 8,39$ & $25,83 \pm 9,19$ \\
Pantorilla & $26,16 \pm 5,39$ & $21,16 \pm 5,06$ \\
Muslo anterior & $34,08 \pm 6,07$ & $29,08 \pm 5,95^{*}$ \\
Supra espinal & $22,08 \pm 5,63$ & $20,25 \pm 5,56$ \\
$\sum 7$ pliegues & $167,06 \pm 8,71$ & $145,23 \pm 8,9^{* *}$ \\
\hline
\end{tabular}

Media \pm Desviación Estándar (DE). p<0,05. vas, aunque se observa una leve mejoría en posttest.

En la Tabla V se muestran los resultados comparativos pre-test y post-test en la fuerza muscular de las poblaciones en estudio (Media \pm Desviación Estándar). Se observa un aumento significativo de la fuerza en los músculos pectoral mayor, bíceps braquial y latísimo del dorso $(\mathrm{p}<0,001$ y $\mathrm{p}<0,05)$.

En la Figura 1 se muestra la somatocarta de las jóvenes al inicio del programa (círculo negro) y al final del programa (asterisco), con un claro componente endomórfico. La Figura 2 muestra el registro fotográfico durante la aplicación del programa de ejercicios acuático-aeróbicos.

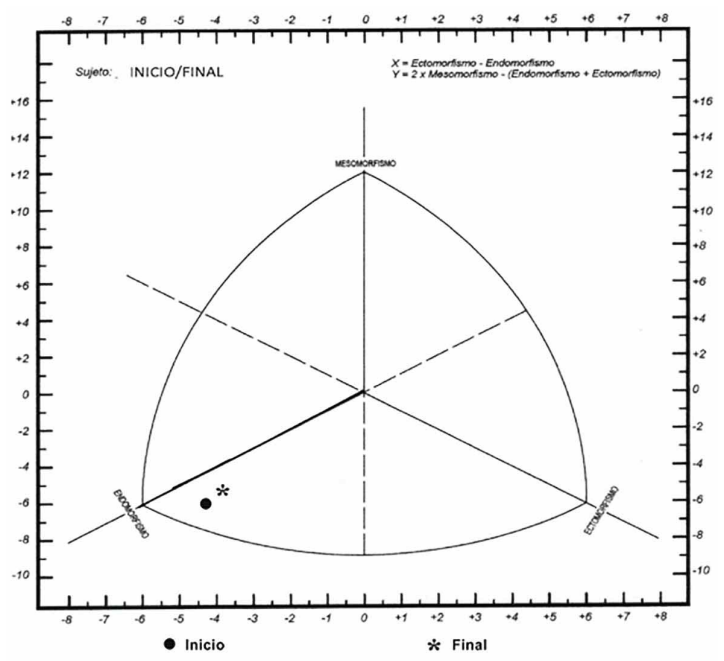

Fig. 1. Somatocarta de las jóvenes de Arica, Chile al Inicio (círculo negro) y final (asterisco) de la aplicación del programa acuático (Carter, 2002).

Tabla III. Mediciones de los perímetros y diámetros corporales de las jóvenes en estudio en el pre-test y el post-test.

\begin{tabular}{llcr}
$\begin{array}{l}\text { Mujeres 16 - 18 años } \\
\mathbf{n = 1 2}\end{array}$ & Brazo relajado & $\begin{array}{c}\text { Pre-Test } \\
\text { (Media } \pm \text { DE) }\end{array}$ & $\begin{array}{c}\text { Post-Test } \\
\text { (Media } \pm \text { DE) }\end{array}$ \\
\hline Perímetros (cm) & Brazo flexionado & $25,41 \pm 3,59$ & $25,99 \pm 3,61$ \\
& Antebrazo & $27,03 \pm 3,23$ & $27,58 \pm 3,29$ \\
& Cintura & $21,61 \pm 1,64$ & $21,85 \pm 1,91$ \\
& Cadera & $80,54 \pm 12,88$ & $78,83 \pm 10,76$ \\
& Pantorrilla & $90,07 \pm 8,2$ & $99,50 \pm 8,43$ \\
Diámetros $(\mathbf{c m})$ & Húmero & $33,97 \pm 2,80$ & $32,91 \pm 3,01$ \\
& Fémur & $5,17 \pm 0,27$ & $5,17 \pm 0,27$ \\
& & $6,98 \pm 0,64$ & $6,98 \pm 0,60$ \\
\hline
\end{tabular}

Media \pm Desviación Estándar (DE). 


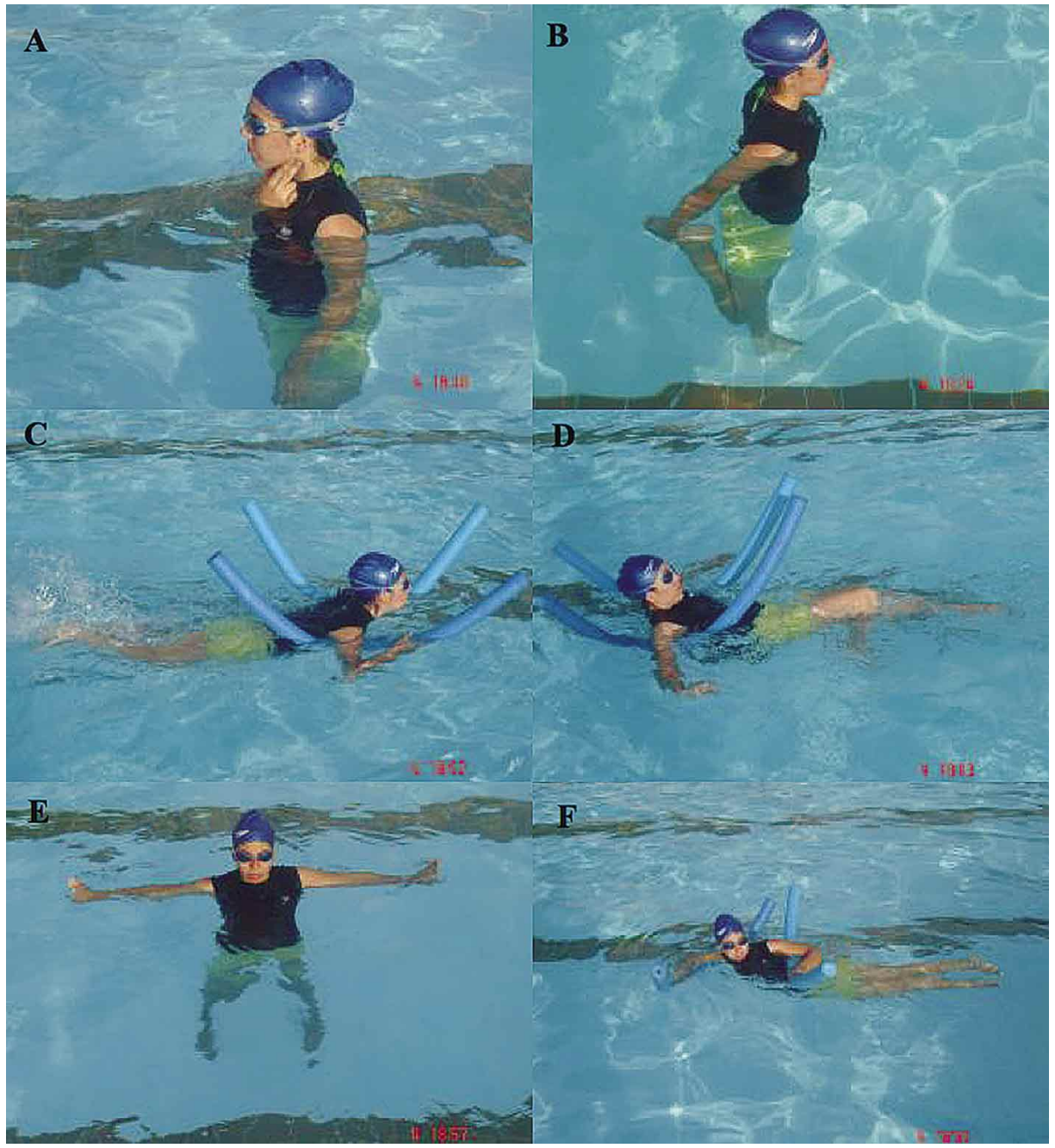

Fig. 2. Registro fotográfico del programa de ejercitación acuático aeróbico. A: Registro del pulso basal. B: Elongación general en el agua, posición vertical. C: Pataleo Crol frontal y dorsal. Posición del horizontal. D: Pataleo piernas en bicicleta, posición frontal y dorsal. E: Piernas en bicicleta. Brazos: Empuje lateral, posición vertical. F: Abdominal lateral, posición horizontal.

Tabla IV. Componentes del somatotipo en las mujeres al inicio y al final del programa de ejercicios acuáticoaeróbicos.

\begin{tabular}{|c|c|c|c|c|c|}
\hline \multirow[b]{2}{*}{$\begin{array}{l}\text { Mujeres 16-18 años } \\
n=12\end{array}$} & \multicolumn{5}{|c|}{ Somatotipo } \\
\hline & Endomorfo & Mesomorfo & Ectomorfo & $\mathbf{X}$ & $\mathbf{Y}$ \\
\hline $\begin{array}{l}\text { Pre-Test } \\
(\text { Media } \pm D E)\end{array}$ & $6,19 \pm 1,52$ & $1,4 \pm 1,04$ & $1,82 \pm 1,08$ & $-4,4 \pm 2,4$ & $-5,2 \pm 2,45$ \\
\hline $\begin{array}{l}\text { Post-Test } \\
(\text { Media } \pm \mathrm{DE})\end{array}$ & $5,65 \pm 1,65$ & $1,4 \pm 1,2$ & $1,7 \pm 1,05$ & $-3,9 \pm 2,6$ & $-4,4 \pm 2,46$ \\
\hline
\end{tabular}

Media \pm Desviación Estándar (DE). 


\begin{tabular}{lcc}
\hline $\begin{array}{l}\text { Mujeres 16 - 18 años } \\
\mathbf{n}=\mathbf{1 2}\end{array}$ & $\begin{array}{c}\text { Pre-Test } \\
\text { (Media } \pm \mathbf{D E} \text { ) }\end{array}$ & $\begin{array}{c}\text { Post-Test } \\
\text { (Media } \pm \mathbf{D E} \text { ) }\end{array}$ \\
\hline Pectoral mayor & $15,83 \pm 4,17$ & $23,16 \pm 5,33^{* *}$ \\
Tríceps braquial & $8,25 \pm 1,76$ & $9,25 \pm 1,60$ \\
Bíceps braquial & $8,66 \pm 1,77$ & $12,58 \pm 2,65^{*}$ \\
Latísimo del dorso & $8,58 \pm 3,05$ & $12,25 \pm 2,65^{*}$ \\
Recto abdominal & $15,08 \pm 9,52$ & $22,00 \pm 13,38$ \\
Abdominal corto & $53,16 \pm 10,13$ & $60,58 \pm 14,08$ \\
Glúteo máximo & $28,16 \pm 6,49$ & $31,43 \pm 5,67$ \\
$\sum 7$ & $137,72 \pm 5,27$ & $158,67 \pm 6,48^{* *}$
\end{tabular}

Tabla V. Efecto del programa de ejercicios acuático-aeróbicos sobre el desarrollo de la fuerza muscular $(\mathrm{kg})$ en las participantes. Se observa un incremento significativo en la fuerza de los músculos pectoral mayor, bíceps braquial, latísimo del dorso y en la sumatoria de los siete músculos en estudio $\left(\sum 7\right)$, al finalizar el programa.

\section{DISCUSIÓN}

En las últimas décadas los ejercicios acuáticos se han convertido en uno de los mejores tipos de actividades corporales con grandes beneficios cardiovasculares y metabólicos, provocando cambios en la fuerza muscular, la flexibilidad y mejoras significativas en la composición corporal (Barbosa et al.). Los cambios en la composición corporal de los individuos se asocian directamente con la actividad física y la adecuada nutrición (Minsal, 2004; Pantelic et al.).

La cineantropometría se ha considerado como la forma de interrelacionar la estructura y el funcionamiento del cuerpo humano (Marfell-Jones et al.; Moreno et al., 2006).

Las mediciones de los pliegues subcutáneos, el índice de masa corporal y el índice cintura/cadera permiten una aproximación sencilla de la distribución de los diferentes componentes corporales (Garrido-Chamorro et al., 2012; Díaz \& Espinoza-Navarro; Moreno, 2012). La composición corporal y el somatotipo, se relaciona directamente con el nivel de condición física y el rendimiento al igual que con la obesidad y las enfermedades cardiovasculares (Flegal et al., 2009; Carter; Lizana Arce et al., 2012).

Pantelic et al., determinaron que programas de ejercicios con danza aeróbica, disminuyen el tejido graso subcutáneo y la composición corporal en mujeres jóvenes. Similares resultados observan Gubiani \& Pires Neto (2006), con una reducción significativa de los pliegues cutáneos, la sumatoria de los pliegues regional, el porcentaje de grasa corporal y del peso corporal. Los resultados obtenidos por estos autores son similares a los observados en este estudio.

Lizana Arce et al., muestran que el somatotipo de mujeres adolescentes de Valparaíso, Chile se orientan ha- cia un biotipo predominantemente endomórfico con disminución del componente mesomórfico. Estos resultados son coincidentes con las evaluaciones obtenidas en las jóvenes de Arica.

El entrenamiento acuático tiene un amplio potencial la promoción y prevención de la salud en todas las personas, sin embargo sigue siendo una modalidad poco utilizada (Pöyhönen et al.; Tsourlou et al., 2006; Becker). Los estudios realizados en población chilena permiten apoyar los objetivos sanitarios planteados en la "Estrategia Nacional de Salud", para la década 2011-2020, de aumentar en un $30 \%$ la práctica de la actividad física en adolescentes y jóvenes chilenos (Minsal).

\section{CONCLUSIONES}

El programa de ejercicios acuático-aeróbicos, aplicado a las jóvenes de Arica, Chile, provocaron un efecto positivo, con aumento significativo en la fuerza muscular y una disminución significativa en los pliegues cutáneos. La aplicación de este tipo de programas acuáticos, otorgan múltiples beneficios para la salud y en la mejora de la calidad de vida.

\section{AGRADECIMIENTOS}

El grupo de trabajo agradece la colaboración del Dr. Jorge Díaz Gamboa en la supervisión de la medición de los parámetros corporales. Este trabajo fue subvencionado por el proyecto UTA Mayor $\mathrm{N}^{\circ}$ 5750-12 de la Universidad de Tarapacá, Arica-Chile. 
PINO-REYNALS, A.; ESPINOZA-NAVARRO, O.; DE ARRUDA, M. \& URIZAR-ARAYA, C. Body composition and somatotype in women between 16 and 18 years of age: Effect of an aquatic-aerobics program on the improvement of muscular strength. Int. $\boldsymbol{J}$. Morphol., 33(4):1213-1219, 2015.

SUMMARY: Aquatic activities are a clear alternative to programs of terrestrial muscle strengthening. The aim of this study was to determine the effect of aquatic-aerobics exercises on folds and body diameters and improving muscle strength in young women. Twelve women between 16-18 years old, sedentary, intentionally selected were subjected to an aquatic-aerobics exercises program of 18 sessions, developed at the Olympic Swimming Pool in Arica-Chile. The results show that young women have an overweight BMI, with a predominantly endomorph somatotype. The body folds of anterior thigh and calf decreased significantly at the end of program implementation, similar behavior expressed in the sum of these ( 27 : of $167.06 \pm 8.71 \mathrm{~mm}$ to $145.23 \pm 8.9 \mathrm{~mm}$ in pretest). Muscle strength was significantly increased in the pectoralis major, latissimus dorsi and brachial biceps muscles. However, this increase is more significant ( $\mathrm{p}<0.001)$ in the sum of muscles evaluated (?7: of $137.72 \pm 5.27 \mathrm{~kg}$ to $158.67 \pm 6.48 \mathrm{~kg}$ after the program). It is concluded that aquaticaerobics exercise programs applied to young women, have a positive effect, with significant increases in muscle strength and a significant decrease in skin folds. The implementation of these programs provides multiple benefits for health and improved quality of life.

KEY WORDS: Aquatic exercise; Muscle strength; Swimming; Chile.

\section{REFERENCIAS BIBLIOGRÁFICAS}

Barbosa, T. M.; Marinho, D. A.; Reis, V. M.; Silva, A. J. \& Bragada, J. A. Physiological assessment of head-out aquatic exercises in healthy subjects: a qualitative review. J. Sports Sci. Med., 8(2):179-89, 2009.

Becker, B. E. Aquatic therapy: scientific foundations and clinical rehabilitation applications. PMR, 1(9):859-72, 2009.

Brunetto, A. F.; Paulin, E. \& Yamaguti, W. P. S. Comparação entre a escala de Borg modificada e a escala de Borg modificada análogo visual aplicadas em pacientes com dispnéia. Rev. Bras. Fisioter., 6(I):41-5, 2002.

Carter, J. E. L. The Heath-Carter anthropometric somatotype. Instruction manual. San Diego, San Diego State University, 2002.

Colado, J. C. \& Triplett, N. T. Monitoring the intensity of aquatic resistance exercises with devices that increase the drag force: An update. J. Strength Cond. Res., 31(3):94-100, 2009.

Díaz, J. \& Espinoza-Navarro, O. Determination of fat mass percentage by measuring body circumferences, weight and height: A validation study. Int. J. Morphol., 30(4):1604-10, 2012.

Edwards, S. Corazón Inteligente. Madrid, Dorleta, 1996.

Espinoza-Navarro, O. \& Valle, S. Body composition and the effect of an auxiliary force program to prevent injuries in femoral quadriceps, hamstrings and biceps femoris muscles in young college players. Int. J. Morphol., 32(3):1095-100, 2014.

Flegal, K. M.; Shepered, J. A.; Looker, A. C.; Graubard, B. I.; Borrud, L. G.; Ogden, C. L.; Harris, T. B.; Everhart, J. E. \& Schenker, N. Comparisons of percentage body fat, body mass index, waist circumference, and waist-stature ratio in adults. Am. J. Clin. Nutr., 89(2):500-8, 2009.

1218
Garrido-Chamorro, R.; Sirvent-Belando, J. E.; González-Lorenzo, M.; Blasco-Lafarga, C. \& Roche, E. Skinfold Sum: Reference values for top athletes. Int. J. Morphol., 30(3):8039, 2012.

Gubiani, G. L. \& Pires Neto, C. S. Effects of a step training program on anthropometric and body composition variables of female college students. Braz. J. Kinanthropom. Hum. Performance, 1(1):89-95, 2006.

International Society for Advancements of Kinanthropometry (ISAK). International Standards for Anthropometric Assessment. Underdale, ISAK, 2001.

Lizana Arce, P.; Almagiá Flores, A.; Simpson Lelievre, C.; Ivanovic Marincovic, D.; Binvignat Gutiérrez, O. \& Berral de la Rosa, F. Changes of somatotype in high school students, V region, Chile: 1985-2010. Nutr. Hosp., 27(1):270-5, 2012.

Marfell-Jones, M.; Olds, T.; Stewart, A. \& Carter, L. International standards for anthropometric assessment. Potchefstroom, ISAK, 2006.

Ministerio de Salud de Chile (Minsal). Estrategia Nacional de Salud. Para el cumplimiento de los objetivos sanitarios de la Década 2011-2020. Santiago de Chile, Minsal, 2010. Disponible en: http://web.minsal.cl/portal/url/item/ c4034eddbc96ca6de0400101640159b8.pdf

Moreno, J. A. \& Gutiérrez Sanmartín, M. Bases metodológicas para el aprendizaje de las actividades acuáticas educativas. Barcelona, INDE Publicaciones, 1998.

Moreno, G. M. Definición y clasificación de la obesidad. Rev. Med. Clin. Condes, 23(2):124-8, 2012. 
PINO-REYNALS, A.; ESPINOZA-NAVARRO, O.; DE ARRUDA, M. \& URIZAR-ARAYA, C. Composición corporal y somatotipo de mujeres de entre 16 a 18 años: efecto de un programa acuático-aeróbico en el mejoramiento de la fuerza muscular. Int. J. Morphol., 33(4):1213-1219, 2015.

Moreno, L. A.; Mesana, M. I.; González-Gross, M.; Gil, C. M.; Fleta, J.; Wärnberg, J.; Ruiz, J. R.; Sarría, A.; Marcos, A.; Bueno, M. \& AVENA Study Group. Anthropometric body fat composition reference values in Spanish adolescents. The AVENA Study. Eur. J. Clin. Nutr., 60(2):191-6, 2006.

Pantelic, S.; Milanovic, Z.; Sporis, G. \& Stojanovic-Tosic, J. Effects of a twelve-week aerobic dance exercises on body compositions parameters in young women. Int. J. Morphol., 31(4):1243-50, 2013 .

Pöyhönen, T.; Sipilä, S.; Keskinen, K. L.; Hautala, A.; Savolainen, J. \& Mälkiä, E. Effects of aquatic resistance training on neuromuscular performance in healthy women. Med. Sci. Sports Exerc., 34(12):2103-9, 2013.

Tsourlou, T.; Benik, A.; Dipla, K.; Zafeiridis, A. \& Kellis, S. The effects of a twenty-four-week aquatic training program on muscular strength performance in healthy elderly women. $J$. Strength Cond. Res., 20(4):811-8, 2006.

Unidad de Nutrición del Ministerio de Salud \& Consejo Asesor en Nutrición. Norma técnica de evaluación nutricional del niño de 6 a 18 años. Rev. Chil. Nutr., 31(2):128-37, 2004.

World Health Organization (WHO). Estrategia mundial sobre régimen alimentario, actividad física y salud. Ginebra, $57^{\circ}$ Asamblea Mundial de la Salud, 2004. Disponible en: http:// apps.who.int/gb/ebwha/pdf_files/WHA57/A57_R17-sp.pdf 